\title{
Advances in AAV-mediated gene transfer for the treatment of inherited disorders
}

\author{
Markus Hildinger ${ }^{1}$ and Alberto Auricchio*,2 \\ ${ }^{1}$ Boston Consulting Group, Exchange Place, Boston, MA, USA; ${ }^{2}$ Telethon Institute of Genetics and Medicine (TIGEM), \\ Via Pietro Castellino 111, Napoli 80131, Italy
}

The holy grail of gene therapy is the cure of genetic diseases. To achieve this goal, a vector system is desirable that offers a high level of safety combined with clinical efficacy and versatility in terms of potential applications. Gene therapy vectors based on recombinant adeno-associated viruses (AAVs) meet all of these criteria: They are nonpathogenic, devoid of viral coding sequences, and mediate long-term gene expression in the absence of an immune or inflammatory response. Moreover, with the recent discovery of novel AAV serotypes, there is now one preferred serotype for nearly every organ or tissue to target. Thus, AAV gene therapy vectors are increasingly becoming the vectors of choice for the treatment of inherited disorders.

European Journal of Human Genetics (2004) 12, 263-271. doi:10.1038/sj.ejhg.5201153

Published online 14 January 2004

Keywords: adeno-associated viral vectors; gene therapy; inherited disorders

\section{Biology of adeno-associated viruses (AAVs)}

$\mathrm{AAV}$ is a small, nonpathogenic virus of the parvoviridae family. Compared to other members of its family, AAV depends on a helper virus (eg, adenovirus or herpes virus) for replication, lending to its classification as dependovirus. ${ }^{1,2}$ The virus was coined 'adeno-associated', as it was first discovered as 'impurity' in electron microscopic analyses of adenoviruses, that is, the name does not infer any specific biologic similarity or relationship between the two - apart from the fact that adenovirus among other viruses can function as a helper virus for AAV replication.

The approximately $5 \mathrm{~kb}$ genome of AAV consists of single-stranded DNA of either plus or minus polarity. The ends of the genome form short inverted terminal repeats (ITRs), which can fold into hairpin structures and serve as the origin of viral DNA replication. The ITRs are the only

*Correspondence: $\operatorname{Dr}$ A Auricchio, TIGEM, Via Pietro Castellino 111, Napoli 80131, Italy. Tel: + 1139081 6132229; Fax: + 1139081 6132351; E-mail: auricchio@tigem.it

Received 28 August 2003; revised 18 November 2003; accepted 20 November 2003 cis elements required for replication and packaging. ${ }^{3,4}$ The genome is packaged within a nonenveloped, icosahedral capsid of approximately $20 \mathrm{~nm}$ diameter. ${ }^{5}$

To date, at least eight serologically distinct AAVs have been identified and isolated from humans or primates ${ }^{4,6-10}$ (Table 1), although the genome from several additional AAVs has been isolated from primate tissues. ${ }^{14}$ The most extensively studied of these isolates are AAV type 2 (AAV2), the only AAV serotype in active clinical trials, and AAV type 5 (AAV5). Whereas the different serotypes differ in terms of tissue tropism, the genomes can be cross-packaged into heterologous capsids in general, ${ }^{15}$ with a few exceptions, that is, AAV5 genomes into AAV2 capsids. ${ }^{16}$

The AAV genome harbors two open reading frames $(\mathrm{ORFs})^{2}$. The left ORF encodes the nonstructural Rep proteins: Rep40, Rep52, Rep68 and Rep78, which are involved in regulation of replication and transcription (the numbers reflect the respective molecular weights). Rep68/78 have also been shown to possess ATP-binding activity as well as DNA and RNA helicase activities. The larger Rep proteins (Rep68/78) are transcribed from the p5 promoter, the shorter Rep proteins (Rep40/52) from the 
Table 1

\begin{tabular}{|c|c|c|c|c|c|}
\hline Vector & Isolation & Receptor & Column purification & $\begin{array}{c}\text { Cross-packaging } \\
\text { with AAV2 }\end{array}$ & Preferred tissue/cell tropism \\
\hline AAV1 (AAV6) & Cell line & $\mathrm{n} / \mathrm{a}$ & $\mathrm{n} / \mathrm{a}$ & Yes & Muscle; liver; lung; eye \\
\hline AAV2 & Cell line & FGFR1; heparin & Heparin & Yes & Muscle; brain; liver; eye \\
\hline AAV3 & Cell line & $\mathrm{n} / \mathrm{a}$ & $\mathrm{n} / \mathrm{a}$ & Yes & $\mathrm{n} / \mathrm{a}$ \\
\hline AAV4 & Cell line & Sialic acid & $\mathrm{n} / \mathrm{a}$ & Yes & Brain \\
\hline AAV5 & Human lesion & PDGFR; sialic acid & Mucin & Yes & Brain; muscle; liver; lung; eye \\
\hline AAV6 (AAV1) & Cell line & $\mathrm{n} / \mathrm{a}$ & $\mathrm{n} / \mathrm{a}$ & Yes & Muscle; liver; lung; eye \\
\hline AAV7 & Monkey & $\mathrm{n} / \mathrm{a}$ & $\mathrm{n} / \mathrm{a}$ & Yes & Muscle; liver \\
\hline AAV8 & Monkey & $\mathrm{n} / \mathrm{a}$ & $\mathrm{n} / \mathrm{a}$ & Yes & Liver \\
\hline
\end{tabular}

Bold italic: highest level of gene expression of all AAV serotypes.

n/a: not available; FGFR 1: fibroblast growth factor receptor 1; PDGFR: platelet-derived growth factor receptor.

p19 promoter (the number indicates the relative position within the AAV genome). Alternative splicing then leads to the generation of the corresponding variants.

The right ORF encodes the three capsid proteins VP1, 2 and 3, which are all transcribed from the same promoter (p40 promoter). Their coding regions are overlapping, and the different capsid proteins are thus generated by alternative splicing and by the use of an unusual start codon (ACG). These capsid proteins form the icosahedral virion particle in the ratio of 1:1:10 (VP1:VP2:VP3).

It has been shown that the wild-type AAV2 genome preferentially integrates into a region of the $\mathrm{q}$ arm of human chromosome 19 (AAVS1) - catalyzed by the activity of the Rep proteins. ${ }^{17-19}$ Specific integration sites for the other AAV serotypes have not yet been identified. The extent to which recombinant genomes are able to integrate in the absence of Rep functions is still in discussion. However, recently, Mark Kay's group at Stanford University has shown that recombinant AAV2 vectors preferentially integrate in transcriptionally active chromatin regions in hepatocytes ${ }^{20,21}$ - although overall integration frequency has been low. Whether this is a general phenomenon, or specific for liver or AAV2, has to be further investigated.

In order to make use of AAV for gene therapy purposes, the gene of interest replaces the viral Rep and Cap ORFs; that is, all but the ITR sequences are removed from the gene therapy vector. Recombinant virus particles are produced by providing the packaging cell with the essential Rep and Cap functions in trans as well as the necessary viral helper functions.

\section{What makes AAV attractive for gene therapy?}

The following features have made AAV an attractive vector for gene therapy:

(1) AAV vector particles have favorable characteristics: They are nonpathogenic, noninflammatory, heat stable and resistant to solvents and changes in $\mathrm{pH}$ and temperature. This distinguishes recombinant AAV vectors from many other viral vector systems such as retro/lentiviral and adenoviral vectors. ${ }^{22}$
(2) As mentioned above, AAV vectors are devoid of all viral genes and only retain about 300 nucleotides of viral sequence in the form of nontranscribed ITRs. This greatly improves the safety of AAV vectors for human clinical applications by reducing the risk of recombination with wild-type virus. Furthermore, the lack of viral coding sequences extends the duration of gene expression as no viral gene products are expressed in target cells, which reduces the risk of eliciting a cellular immune response.

(3) AAV vectors possess a broad host range and transduce both dividing and nondividing cells in vitro and in vivo. With the recent discovery of novel AAV serotypes, the universe of potential target organs, tissues and cells will expand even further. ${ }^{7,10,14}$

(4) The most promising and distinguishing feature of AAV vectors is the fact that they maintain (over several years) high levels of gene expression in vivo in the absence of a significant immune response to the transgene product. This is a major requirement for gene therapy applications to treat and cure inherited disorders. ${ }^{23}$

However, there are also a few drawbacks in using AAV vectors for gene therapy applications:

(1) The AAV genome allows for transfer of gene expression cassettes of up to approximately $4.5 \mathrm{~kb}{ }^{4}$ Whereas strategies have been developed to overcome this limitation based on trans-splicing ${ }^{24,25}$ and intermolecular recombination, ${ }^{26}$ those approaches still suffer from low efficiency resulting in decreased levels of gene expression. Especially in instances where a high level of gene expression is required (eg, expression of Factor VIII), these methods might not suffice to provide a therapeutic benefit in their current form.

(2) Onset of gene expression after AAV transduction is slow - especially compared to other viral vector systems such as adenoviral vectors. This is due to the fact that AAV genomes are single-stranded and have to be converted into double-stranded DNA before gene expression can be initiated. ${ }^{2}$ Depending on the serotype and target organ, it can take up to 3 months 
to achieve an appropriate level of gene expression. ${ }^{23}$ However, also in this instance, strategies have been developed to overcome this limitation, ranging from co-administration of chemicals favoring the conversion into double-stranded $\mathrm{DNA}^{27}$ to vector constructs that are internally complementary; that is, that can anneal intramolecularly and generate double-stranded DNA. ${ }^{28}$ The major disadvantage of the latter approach is the further reduction in maximum gene size to be transferred, as one has to incorporate the gene twice into the vector (in its sense and antisense orientation).

(3) In one 2001 study, $^{29}$ preclinical data from an animal model for mucopolysaccharidosis VII (MPS VII), a lysosomal storage disease caused by mutations in the $\beta$-glucuronidase gene, suggested a possible association between AAV2 vector gene transfer and tumorigenesis: An AAV2 vector encoding the human GUSB gene was intravenously injected into neonatal mice. After administration, the vector was detected in multiple cell types and produced sufficient amounts of $\beta$-glucuronidase so that the treated mice showed improvements in the disease symptoms for over a year. As the mice were studied, hepatomas and angiosarcomas were detected in six of the vector-treated mice. So far, the results have not yet been reproduced, and the cause for the malignancies is still somewhat unclear.

(4) Mark Kay's group at Stanford University has shown that recombinant AAV2 vectors preferentially integrate into transcriptionally active chromatin regions in hepatocytes. $^{20,21}$ The group cloned a total of 29 integration sites, of which 21 were within genes. However, the overall integration frequency of rAAV2 vectors is extremely low, and there still remains the question whether this is a general phenomenon, or specific for liver (or the hepatocyte in vivo model used) or AAV2. On the other hand, it has been published that certain recombination events require transcription, and recombination might be involved in rAAV2 integration. ${ }^{30,31}$
Despite existing limitations and concerns that still need to be addressed and resolved, gene transfer vectors based on recombinant AAVs still represent one of the most promising gene therapy systems and gain increasing popularity. Thus, it comes at no surprise that the first gene therapy applications based on rAAV vectors have entered the clinical stage.

\section{Clinical applications of AAV vectors}

Interest in the use of AAV vectors for clinical gene transfer has been increasing due to its favorable features as a vector system. In 1994, the Recombinant DNA Advisory Committee (RAC) reviewed the first AAV vector protocol. This protocol and several subsequent studies are investigating gene transfer for the treatment of cystic fibrosis (CF). Other clinical research proposals have focused on hemophilia B, limb girdle muscular dystrophy, amyotrophic lateral sclerosis and Canavan disease (CD) among others (Table 2).

Whereas a general proof-of-concept in terms of successful nucleic acid transfer in the absence of a significant immune response has been achieved, the therapeutic benefit to patients has so far been limited. ${ }^{32-38}$ Two main causes (apart from vector dosage) can be identified:

(1) The amount of rAAV2-mediated gene expression was not high enough to provide a long-term therapeutic benefit, for example, in the case of hemophilia B. ${ }^{39}$

(2) The tissue/ cell tropism of rAAV2 was insufficient to transduce a high enough percentage of target cells, for example, in the case of $\mathrm{CF}^{33}$

In reviewing past studies and before making a final judgement on the usefulness of AAV for human applications, one should not forget that these first trials were conducted with first-generation AAV2 vectors. AAV vector development, production and expression cassette design have improved significantly in the meantime, be it the use of alternative AAV serotypes to increase tissue and

Table 2

\begin{tabular}{|c|c|c|}
\hline Indication & Gene & Investigator \\
\hline$\alpha 1$-antitrypsin deficiency & $\alpha 1$ antitrypsin & Terrence R Flotte \\
\hline Amyotrophic lateral sclerosis & EAAT2 & Matthew J During \\
\hline Canavan disease & Aspartoacylase & Paola Leone \\
\hline Cystic fibrosis & CFTR & Pamela L Zeitlin \\
\hline Cystic fibrosis & CFTR & Phyllis Gardner \\
\hline Cystic fibrosis & CFTR & Richard B Moss \\
\hline Cystic fibrosis & CFTR & Isabel Virella-Lowell \\
\hline Hemophilia B & Factor IX & Cathrine S Manno \\
\hline Hemophilia B & Factor IX & Bertil Glader \\
\hline Muscular dystrophy & Sarcoglycans & Jerry Mendell \\
\hline Parkinson's disease & Glutamic acid decarboxylase & Matthew J During \\
\hline
\end{tabular}

The Journal of Gene Medicine (Clinical Trial Database; August 2003).

CFTR: Cystic fibrosis transductance regulator; EAAT2: excitory amino acid transporter 2. 
cell tropism, column purification of vector instead of cesium chloride density centrifugation, ${ }^{40,41}$ or the inclusion of post-transcriptional regulatory elements into expression cassettes to improve gene expression. ${ }^{42}$ Thus, a final word has not yet been spoken.

Let us first review previous and ongoing clinical trials, and then take a look at the promising preclinical strategies for the treatment of genetic disorders.

\section{AAV2-based gene therapy for the treatment of CF}

$\mathrm{CF}$ is the most common autosomal recessive disorder in caucasians, with a frequency of about 1 in 2500 live births. The disease is caused by mutations in the CF transmembrane conductance regulator (CFTR). This ABC-type chloride channel is primarily found in epithelial cells in the airways, pancreas, salivary glands and sweat glands, where it helps regulating the intracellular chloride concentration. When CFTR functionality is abolished through mutations, thick, sticky mucus builds up in the lungs and digestive system. This abnormal mucus inhibits normal immune defense in the lungs and results in frequent infections, chronic inflammation and scarring. With time, lung function deteriorates, leading to a loss of respiratory function and death in the end in the second or third decade of life. No effective treatment has been discovered so far. $^{43}$

Given that $\mathrm{CF}$ is a monogenetic disease and does not require tight regulation or high levels of gene expression, the disease was one of the first genetic disorders to be targeted with gene therapy. The main hurdle in genetically treating CF is targeting the right cells within the lung. Moreover, as the gene is relatively large $(\sim 4.4 \mathrm{~kb})$, it is reaching the AAV2 packaging capacity. Targeted genetics tried to overcome the latter limitation by constructing a promoter-less AAV2 vector construct (tgAAVCF), where gene expression is driven by residual ITR promoter function. ${ }^{33,44-47}$

In a Phase II trial, whose results were published in $2002,{ }^{33}$ tgAAVCF was administered to 23 patients. In this double-blind, randomized, placebo-controlled study, each patient was administered a dose of 100000 replication units of tgAAVCF into one maxillary sinus (replication units defer from genomic copies in that they measure the number of infective vector particles: In a typical AAV preparation, only one out of 10-100 particles is infective; thus, a genomic copy titer overestimates the amount of infective or replication units); the contralateral maxillary sinus received a placebo treatment as internal control.

The tgAAVCF administration was well tolerated, without adverse respiratory events or increase in the titer of AAV neutralizing antibodies in the serum. Furthermore, there were no signs of enhanced inflammation in sinus histopathology. However, neither the primary efficacy end point (rate of relapse of clinically defined, endoscopically diagnosed recurrent sinusitis) nor several secondary end points (sinus transepithelial potential difference, histopathology, sinus fluid interleukin-8 measurements) achieved statistical significance when comparing treated to control sinuses within patients.

Thus, whereas rAAV2 administration to the lung seems to be safe and well tolerated, there has been little support of clinical efficacy. The reason for this can be three-fold:

(1) AAV2-mediated CFTR gene expression induced an immune response leading to the destruction of transduced lung cells,

(2) tgAAVCF expression is too low,

(3) AAV2 does not transduce lung epithelial cells to a therapeutically relevant level.

Most evidence points to (3), and it will be interesting to see if higher dosages of tgAAVCF can compensate for lack of cell tropism. In any case, targeted genetics continues its efforts and starts a 100-patient Phase IIb study (a phase IIb is a second, extended phase II study) with tgAAVCF. Study participants will receive two doses of $10^{13}$ DNAse-resistant particles (DRP; the terms DNA-resistant particles, genomic particles or vector genomes are used interchangeably in the literature with genomic copies or particles) of tgAAVCF, administered via a nebulizer at days 0 and 30 of the study. The primary read-out will take place after 90 days.

\section{AAV2-based gene therapy for the treatment of hemophilia $B$}

Hemophilia B (also called 'Christmas disease' after Stephen Christmas, the first person diagnosed with the disease) is an X-chromosomal, inherited bleeding disorder caused by mutations in the Factor IX gene. One out of 34500 men is affected by this disease. ${ }^{48}$

Factor IX is involved in blood clotting; if its function is impaired, prolonged bleeding results, which can lead to permanent joint and muscle damage in case of uncontrolled internal bleeding. Today, the disease can be managed through administration of recombinant Factor IX. Yet, prophylactic treatment with Factor IX would be desirable. Whereas such a treatment regimen with recombinant protein is feasible in theory, it would be prohibitively expensive. Expression of Factor IX by rAAV-mediated gene transfer might provide a solution.

Hemophilia B reveals several characteristics that make it a good candidate for a gene therapeutic approach:

(1) Only a relatively small amount of protein $(1.5-5 \%$ of normal serum levels; preferentially $3-10 \%)$ is required for phenotypic correction, and there is no need for tight regulation of gene expression - neither in terms of quantity nor in terms of physiologic condition. ${ }^{39}$

(2) The Factor IX cDNA is relatively short $(\sim 1.4 \mathrm{~kb})$, especially compared to the Factor VIII cDNA (Factor VIII is functionally impaired in the more common Hemophilia A), and thus fits easily into an rAAV 
genome, even as a self-complementary expression cassette for faster onset of gene expression.

(3) As Factor IX is a secreted protein, one can target different organs - such as muscle, ${ }^{34}$ liver $^{49}$ or lung $^{50}$ for its expression and secretion into the blood stream.

Thus, the main challenge for the treatment of Hemophilia B is the level of Factor IX gene expression that can be achieved.

Given these favorable characteristics, it is not surprising that Avigen and the Children's Hospital of Philadelphia together with Stanford University Medical Center are conducting clinical studies to evaluate the potential of an AAV2-based vector for the transfer and expression of recombinant Factor IX. Phase I/II clinical trials began at the Children's Hospital of Philadelphia and Stanford University Medical Center in June 1999, where patients were given the vector either by intramuscular injection or hepatic artery infusion.

Intramuscular delivery In December 2000, preliminary results from the intramuscular study branch were presented at the American Society of Hematology meeting: six out of seven patients showed successful transduction of muscle cells. Two of the six low- and medium-dose patients showed elevation of Factor IX protein at levels greater than or equal to $1.0 \%$ of the normal. In addition, two patients exhibited a significant reduction in Factor IX usage for nearly a year and a half.

In July 2002, additional data of a phase I/II open-label, dose-escalation study in eight patients with severe hemophilia B have been published. ${ }^{51}$ The vector was again well tolerated, but an effective dose could not be established even at the highest regimen of $2 \times 10^{12}$ vector genomes $/ \mathrm{kg}$.

Thus, whereas genetic transduction and Factor IX expression after AAV2-based muscle gene transfer could be demonstrated, the amount of Factor IX expressed was not sufficient to mediate a long-term curative effect. The reason for this can be three-fold:

(1) AAV2-mediated Factor IX gene expression induced an immune response leading to the destruction of transduced muscle cells and/or neutralization of circulating recombinant FIX,

(2) AAV2 transduction efficiency in human muscle is lower compared to animal models,

(3) a higher dosage of AAV2 is required to evoke a curative effect.

Most evidence points to (2) and consequently (3) - as demonstrated in trials with hepatic delivery.

Hepatic delivery In the summer of 2001, the first patients received vector via hepatic artery infusion. However, when vector was detected in the semen of a patient, the trial was put on hold for 3 months - until the vector was cleared from the semen. ${ }^{39}$

Preliminary data from this trial were presented in July 2003 at the 19th International Society on Thrombosis and Haemostasis meeting. By this time, six patients had been treated with vector doses ranging from $8 \times 10^{10}$ to $2 \times 10^{12}$ vector genomes $/ \mathrm{kg}$. One subject in the highest dose regimen showed circulating Factor IX levels in the range of $5-12 \%$ for 5 weeks. No factor IX infusions were required during that time period. Unfortunately, levels then dropped within a week to $2.7 \%$.

In summary, hepatic AAV2 gene delivery results in higher levels of Factor IX expression compared to intramuscular injection. However, in both instances, Factor IX levels drop significantly 4-8 weeks upon vector administration. Thus, although the limited number of patients treated in these studies does not allow for final conclusions, it seems that some, but not all patients, benefit from the treatment, but only for a limited amount of time. It has to be seen if the use of alternative serotypes such as AAV1 in muscle or AAV8 in liver will provide improved benefits for patients. Independent of these efficacy results, however, the vector has been proven safe in all study participants so far.

\section{Preclinical applications of AAV vectors}

Many preclinical applications based on AAV vectors for the treatment and cure of genetic disorders have been described. The major areas of investigation are:

(1) Hematological disorders (eg; Hemophilia A, Hemophilia B, PKU),

(2) Neurological disorders (eg; Parkinson's disease; retinitis pigmentosa; Leber congenital amaurosis; amyotrophic lateral sclerosis),

(3) Muscoskeletal disorders (eg; Duchenne muscular dystrophy; Becker muscular dystrophy),

(4) Cardiovascular diseases (eg; congestive heart failure; hyperlipidemia),

(5) Metabolic disorders (eg, diabetes; ornithine transcarbamylase (OTC) deficiency; CD),

(6) Other genetic disorders (eg, CF; $\alpha 1$-Antitrypsin deficiency).

To illustrate the utility of AAV vectors, we will describe three promising approaches that are close to entering human clinical trials in more detail: novel approaches for the treatment of hemophilia $B$, the use of AAV vectors to treat $\mathrm{CD}$, and the application of of AAV vectors to cure Leber congenital amaurosis (type II).

Recent developments in the gene therapeutic treatment of hemophilia B

As mentioned before, AAV2-mediated Factor IX gene therapy offers only limited therapeutic benefit to patients 
with liver gene transfer being superior to muscle gene transfer. However, from the time of the first hemophilia B clinical trial to today, several novel AAV serotypes have been identified and characterized. These novel serotypes show significantly increased reporter gene expression in the muscle and liver compared to AAV2(7). The question now arises if the use of those serotypes will also result in elevated Factor IX expression in animal models, and eventually later in clinical trials.

Muscle-mediated factor IX gene expression In a preclinical study (presented at the 2003 American Society of Gene Therapy meeting), Yoichi Sakata's group injected 10 cynomolgus monkeys intramuscularly with human factor IX-encoding AAV vectors based on serotypes 1, 2 and 5 at a dose of $10^{12}$ vector genomes $/ \mathrm{kg}$. Two of three AAV1 monkeys reached therapeutic levels of human Factor IX (86 and $138 \mathrm{ng} / \mathrm{ml}$, respectively) after 2 weeks. After 4 weeks, serum levels of human factor IX went down and became undetectable in most cases due to the generation of anti-human Factor IX antibodies (although cynomolgus and human Factor IX share 97\% sequence homology). However, this experiment demonstrated the advantage of using an alternative serotype (AAV1) for muscle-mediated expression of Factor IX compared to the AAV2 gold standard in a non-human primate model.

Lung-mediated factor IX gene expression The lung offers a large surface area with good vascularization and immense capacity for solute exchange. Moreover, airway epithelial cells are easily accessed by noninvasive aerosol delivery of vectors. Thus, the lung may be used as a site for systemic delivery of secreted therapeutic proteins - such as Factor IX - into the blood stream via AAV-mediated gene transfer. Following noninvasive administration of a Factor IX-expressing AAV5 vector to murine lung, secretion of functional Factor IX in the blood can be observed corresponding to $1.2-1.8 \%$ of normal Factor IX serum levels in humans. ${ }^{50}$ With further optimization of gene expression - either through improvements in expression cassette design or use of other serotypes - lung-mediated Factor IX expression might offer an interesting alternative to muscle and liver, especially as it does not require surgery, which always represents an additional safety concern for hemophilic patients.

Hepatic factor IX gene expression In another preclinical study (presented at the 2003 American Society of Gene Therapy meeting), James Wilson's group, who cloned most of the novel AAVs, compared AAV2 (the previous gold standard) with AAV5 (with strong gene expression in lung and muscle), AAV7 (which is the most efficient in muscle) and AAV8 (which has 10-20 times higher gene expression in liver compared to any other serotype) in a Hemophilia B mouse model in the context of hepatic Factor IX gene transfer. The highest levels of expression were observed for the AAV8 and AAV7 constructs (as measured by ELISA), with at least 10-fold lower expression for AAV2 and AAV5. Similarly, shortest clotting times were observed in the animals receiving AAV8 and AAV7 (in the 25-40 s range). In the mice injected with AAV8, about $70 \%$ of hepatocytes stained positive for intracellular Factor IX protein 8 months after injection. These results clearly demonstrate the superiority of AAV8 over AAV2 in liver-mediated Factor IX expression. The results could be further substantiated in the Chapel Hill hemophilia B dog model. In this experiment, a dog previously treated with $2.8 \times 10^{12}$ genomic copies (GC) $/ \mathrm{kg}$ of AAV2 expressed Factor IX at $0.7 \%$ of normal levels. Upon administration of $5 \times 10^{12} \mathrm{GC} / \mathrm{kg}$ AAV8, Factor IX levels increased to $16 \%$ of normal levels. No bleeding episode has occurred after AAV8 administration (compared to five episodes after the first AAV2 administration).

In summary, these results demonstrate that AAV8 is a more efficient vector than AAV2 in liver-targeted gene therapy, both in small and large animal models of Hemophilia B, and might be the optimal candidate for a human clinical trial.

\section{AAV-based gene therapy for the treatment of CD}

$\mathrm{CD}$, also known as Van Bogaert-Bertrand disease, is a rare, inherited, monogenetic, autosomal-recessive neurological disorder, characterized by spongiform degeneration of the brain. The cause for this childhood leukodystrophy is the loss of function of the enyzme aspartoacylase (ASPA). ASPA is primarily expressed in cells of the central nervous system, and its lack leads to an intracellular accumulation of $\mathrm{N}$-acetyl-aspartate (NAA). This accumulation interferes with normal myelination, leading to spongiform degeneration of the brain. Symptoms of CD may include mental retardation, loss of previously acquired motor skills, abnormal muscle tone and megalocephaly. Blindness, hearing loss, or paralysis may also occur. No effective treatment currently exists. However, AAV-mediated gene transfer of the ASPA gene into patients' brain has the potential to stop or reverse the course of this otherwise fatal condition. ${ }^{11,52-54}$

To explore the feasibility of this gene-replacement approach, the group of Ron Mandel generated a knockout mouse model and injected the striatum and thalamus of these ASPA-deficient mice with a rAAV2 vector encoding the human ASPA cDNA. ${ }^{55}$ At 3-5 months after vector administration, the presence of ASPA in the CD mouse brain could be detected. The group also evaluated functional effects of rAAV2-mediated ASPA expression by standard histological methods, magnetic resonance spectroscopy (MRS) for in vivo NAA levels, and magnetic resonance imaging of CD mice. Interestingly, the treated animals displayed a significant lack of spongiform degeneration in the thalamus. However, pathology in sites 
unrelated to the injected areas showed no improvement in histopathology, emphasizing the importance of either administering rAAV2 to all affected sites in the brain, or choosing an alternative AAV serotype with wider spread within the brain than AAV2, such as for example AAV5. ${ }^{56}$

Based on these and other data, a study protocol has been submitted to the RAC, ${ }^{11-13}$ proposing the direct injection of approximately $9 \times 10^{15}$ genomic particles of ASPAexpressing rAAV2 into affected regions of the brain of 21 patients via neurosurgery. Successful gene transfer will be evaluated via noninvasive biochemical, radiological, and neurological tests.

This gene transfer study would be the first clinical use of AAV in the human brain and the first instance of viral gene transfer for a neurodegenerative disease.

\section{AAV-based gene therapy for the treatment of Leber congenital Amaurosis type II}

AAV vectors provide a useful means to deliver genes to the eye. The eye is particularly well suited for gene therapeutic applications: It represents an immuno-privileged region and allows for local delivery of a relatively high vector concentration at an overall low vector dosage. Efficient gene delivery to photoreceptors and pigment epithelial cells following subretinal injection of AAV has been achieved in various animal models and with different serotypes. $^{57-59}$

Moreover, the genetic basis of many retinal diseases has been identified in recent years - a prerequisite for a gene therapy treatment. One of these diseases is Leber congentical amaurosis (LCA). LCA accounts for 5\% of inherited retinal disorders. It is a heterogeneous, autosomal-recessive disease, and leads to near total blindness in infancy. Three out of 100000 people are affected, and no effective treatment has been discovered so far. Mutations have been described in the genes for RPE65 (vitamin A metabolism), CRB1 (photoreceptor cell structure), RPGRIP1 (protein trafficking), AIPL1 (protein trafficking), GUCY2D (phototransduction) and CRX (retinal embryonic development), as well as linkage to the LCA3 and LCA5 loci. ${ }^{60-63}$

One of the more frequent forms of LCA, accounting for $12 \%$ of all LCA cases, is caused by mutations in the RPE65 gene (LCA type II). Rpe65 is a microsomal protein with exclusive expression in the retinal pigment epithelium (RPE), and is part of the regeneration of 11-cis Retinol from all-trans Retinol. If the enyzme is defective, Rhodopsin is not regenerated, and all-trans retinyl esters accumulate in inclusion bodies within the RPE. In the end, the rod and cone photoreceptor cells degenerate, leading to complete blindness. $^{62}$

A naturally occurring animal model, the RPE65-/- dog, suffers from early and severe visual impairment similar to that seen in human LCA, and thus represents an excellent large animal model of childhood blindness. Several groups have succeeded in correcting the phenotype through rAAV- mediated RPE65 gene transfer. ${ }^{64,65}$ The first group was the one of Dr Jean Bennett. ${ }^{61,64}$ Her group was able to demonstrate correction at the level of electroretinographic (ERG) recordings and visual behavioral testing. Marked improvements were observed as early as 4 weeks after surgery in affected animals, and are still lasting. This proofof-concept study was the first to show that gene therapy can restore and retain (not only delay the loss of) vision in a large-animal model of human retinopathy. Indeed, one of the dogs of this study (Sir Lancelot) even made it to a congressional hearing, to demonstrate the benefits that gene therapy for genetic disorders can deliver.

Given the extremely promising, reproducible results in a large animal model and the suitability of the eye as an organ for gene therapy, AAV-mediated RPE65 gene transfer for the treatment of LCA Type II might become one of the first truly successful gene therapy applications in terms of safety and efficacy.

\section{Summary}

Despite existing limitations and concerns that still need to be addressed and resolved, gene therapy vectors based on AAVs show great promise for the future. Previous clinical studies - although less convincing in terms of efficacy demonstrated so far the lack of toxicity of this vector system and a technological proof-of-concept. With the current improvements in AAV vector and expression cassette design and the advances in identifying novel AAV serotypes, the efficacy boosts observed in animal models should translate into clinical success in humans. Alternatively, by addressing diseases that are more amenable to AAV gene transfer - be it a target organ with high AAV susceptibility (brain) and/or with the need for less virus (eye) - even current vector systems should prove successful. The next 5-10 years should finally deliver on the promise of gene therapy, and put gene therapy on the therapeutic map.

\section{Acknowledgements}

$A A$ is recipient of fundings from the Italian Ministry of the University and of the Scientific and Technological Research (FIRB Gene Therapy), from the Italian Telethon Foundation, from The Ruth and Milton Steinbach Fund, from the Regione Campania and from the NIH (NEI, 1R01EY015136-01).

\section{References}

1 Schlehofer JR, Ehrbar M, zur Hausen H: Vaccinia virus, herpes simplex virus, and carcinogens induce DNA amplification in a human cell line and support replication of a helpervirus dependent parvovirus. Virology 1986; 152: 110-117.

2 Berns KI: Parvovirus replication. Microbiol Rev 1990; 54: 316-329.

3 Samulski RJ et al: Rescue of adeno-associated virus from recombinant plasmids: gene correction within the terminal repeats of AAV. Cell 1983; 33: 135-143. 
4 Samulski RJ et al: Cloning of adeno-associated virus into pBR322: rescue of intact virus from the recombinant plasmid in human cells. Proc Natl Acad Sci USA 1982; 79: 2077-2081.

5 Xie $\mathrm{Q}$ et al: The atomic structure of adeno-associated virus (AAV-2), a vector for human gene therapy. Proc Natl Acad Sci USA 2002; 99: 10405-10410.

6 Rutledge EA, Halbert CL, Russell DW: Infectious clones and vectors derived from adeno-associated virus (AAV) serotypes other than AAV type 2. J Virol 1998; 72: 309-319.

7 Gao GP et al: Novel adeno-associated viruses from rhesus monkeys as vectors for human gene therapy. Proc Natl Acad Sci USA 2002; 99: 11854-11859.

8 Chiorini JA et al: Cloning of adeno-associated virus type 4 (AAV4) and generation of recombinant AAV4 particles. J Virol 1997; 71: $6823-6833$.

9 Chiorini JA et al: Cloning and characterization of adenoassociated virus type 5. J Virol 1999; 73: 1309-1319.

10 Grimm D, Kay MA: From virus evolution to vector revolution: use of naturally occurring serotypes of adeno-associated virus (AAV) as novel vectors for human gene therapy. Curr Gene Ther 2003; 3: 281-304.

11 Leone $\mathrm{P}$ et al: Global CNS gene transfer for a childhood neurogenetic enzyme deficiency: Canavan disease. Curr Opin Mol Ther 1999; 1: 487-492.

12 Janson C et al: Clinical protocol. Gene therapy of Canavan disease: AAV-2 vector for neurosurgical delivery of aspartoacylase gene (ASPA) to the human brain. Hum Gene Ther 2002; 13: 13911412.

13 Leone $\mathrm{P}$ et al: Aspartoacylase gene transfer to the mammalian central nervous system with therapeutic implications for Canavan disease. Ann Neurol 2000; 48: 27-38.

14 Gao G et al: Adeno-associated viruses undergo substantial evolution in primates during natural infections. Proc Natl Acad Sci USA 2003; 100: 6081-6086.

15 Hildinger $\mathrm{M}$ et al: Hybrid vectors based on adeno-associated virus serotypes 2 and 5 for muscle-directed gene transfer. J Virol 2001; 75: 6199-6203.

16 Grimm D: Production methods for gene transfer vectors based on adeno-associated virus serotypes. Methods 2002; 28: 146-157.

17 Kotin RM et al: Site-specific integration by adeno-associated virus. Proc Natl Acad Sci USA 1990; 87: 2211-2215.

18 Samulski RJ et al: Targeted integration of adeno-associated virus (AAV) into human chromosome 19. EMBO J 1991; 10: 3941-3950.

19 Samulski RJ: Adeno-associated virus: integration at a specific chromosomal locus. Curr Opin Genet Dev 1993; 3: 74-80.

20 Nakai $\mathrm{H}$ et al: AAV serotype 2 vectors preferentially integrate into active genes in mice. Nat Genet 2003; 34: 297-302.

21 Russell DW: AAV loves an active genome. Nat Genet 2003; 34: $241-242$.

22 Wright JF et al: Recombinant adeno-associated virus: formulation challenges and strategies for a gene therapy vector. Curr Opin Drug Discov Devel 2003; 6: 174-178.

23 Fisher KJ et al: Recombinant adeno-associated virus for muscle directed gene therapy. Nat Med 1997; 3: 306-312.

24 Yan $\mathrm{Z}$ et al: Trans-splicing vectors expand the utility of adenoassociated virus for gene therapy. Proc Natl Acad Sci USA 2000; 97: 6716-6721.

25 Reich SJ et al: Efficient trans-splicing in the retina expands the utility of adeno-associated virus as a vector for gene therapy. Hum Gene Ther 2003; 14: 37-44.

26 Halbert CL, Allen JM, Miller AD: Efficient mouse airway transduction following recombination between AAV vectors carrying parts of a larger gene. Nat Biotechnol 2002; 20: 697-701.

27 Yakinoglu AO et al: DNA amplification of adeno-associated virus as a response to cellular genotoxic stress. Cancer Res 1988; 48: 3123-3129.

28 McCarty DM, Monahan PE, Samulski RJ: Self-complementary recombinant adeno-associated virus (scAAV) vectors promote efficient transduction independently of DNA synthesis. Gene Therapy 2001; 8: 1248-1254.

29 Donsante A et al: Observed incidence of tumorigenesis in longterm rodent studies of rAAV vectors. Gene Therapy 2001; 8: $1343-$ 1346.

30 Nickoloff JA: Transcription enhances intrachromosomal homologous recombination in mammalian cells. Mol Cell Biol 1992; 12: 5311-5318.

31 Prado F, Piruat JI, Aguilera A: Recombination between DNA repeats in yeast hpr1delta cells is linked to transcription elongation. EMBO J 1997; 16: 2826-2835.

32 High KA: Adeno-associated virus-mediated gene transfer for hemophilia B. Int J Hematol 2002; 76: 310-318.

33 Wagner JA et al: A phase II, double-blind, randomized, placebocontrolled clinical trial of tgAAVCF using maxillary sinus delivery in patients with cystic fibrosis with antrostomies. Hum Gene Ther 2002; 13: 1349-1359.

34 Herzog RW et al: Muscle-directed gene transfer and transient immune suppression result in sustained partial correction of canine hemophilia B caused by a null mutation. Mol Ther 2001; 4: $192-200$.

35 During MJ et al: Subthalamic GAD gene transfer in Parkinson disease patients who are candidates for deep brain stimulation. Hum Gene Ther 2001; 12: 1589-1591.

36 Fabb SA, Dickson JG: Technology evaluation: AAV factor IX gene therapy, Avigen Inc. Curr Opin Mol Ther 2000; 2: 601-606.

37 Wagner JA et al:: Safety and biological efficacy of an adenoassociated virus vector-cystic fibrosis transmembrane regulator (AAV-CFTR) in the cystic fibrosis maxillary sinus. Laryngoscope 1999; 109: 266-274.

38 Barranger JM, Novelli EA: Gene therapy for lysosomal storage disorders. Expert Opin Biol Ther 2001; 1: 857-867.

39 High KA, Theodore E: Woodward Award. AAV-mediated gene transfer for hemophilia. Trans Am Clin Climatol Assoc 2003; 114: 337-351, (discussion 351-2).

40 Auricchio A et al: A single-step affinity column for purification of serotype-5 based adeno-associated viral vectors. Mol Ther 2001; 4: $372-374$.

41 Auricchio A et al: Isolation of highly infectious and pure adenoassociated virus type 2 vectors with a single-step gravity-flow column. Hum Gene Ther 2001; 12: 71-76.

42 Loeb JE et al: Enhanced expression of transgenes from adenoassociated virus vectors with the woodchuck hepatitis virus posttranscriptional regulatory element: implications for gene therapy. Hum Gene Ther 1999; 10: 2295-2305.

43 Kunzelmann K, Nitschke R: Defects in processing and trafficking of cystic fibrosis transmembrane conductance regulator. Exp Nephrol 2000; 8: 332-342.

44 Flotte TR et al: Expression of the cystic fibrosis transmembrane conductance regulator from a novel adeno-associated virus promoter. J Biol Chem 1993; 268: 3781-3790.

45 Haberman RP, McCown TJ, Samulski RJ: Novel transcriptional regulatory signals in the adeno-associated virus terminal repeat A/D junction element. J Virol 2000; 74: 8732-8739.

46 Flotte TR et al: Phase I trial of intranasal and endobronchial administration of a recombinant adeno-associated virus serotype 2 (rAAV2)-CFTR vector in adult cystic fibrosis patients: a two-part clinical study. Hum Gene Ther 2003; 14: 1079-1088.

47 Aitken ML et al: A phase I study of aerosolized administration of tgAAVCF to cystic fibrosis subjects with mild lung disease. Hum Gene Ther 2001; 12: 1907-1916.

48 Bell B, Canty D, Audet M: Hemophilia: an updated review. Pediatr Rev 1995; 16: 290-298.

49 Snyder RO et al: Persistent and therapeutic concentrations of human factor IX in mice after hepatic gene transfer of recombinant AAV vectors. Nat Genet 1997; 16: 270-276.

50 Auricchio A et al: Noninvasive gene transfer to the lung for systemic delivery of therapeutic proteins. J Clin Invest 2002; 110: 499-504. 
51 Manno CS et al: AAV-mediated factor IX gene transfer to skeletal muscle in patients with severe hemophilia B. Blood 2003; 101: $2963-2972$.

52 Gordon N: Canavan disease: a review of recent developments. Eur J Paediatr Neurol 2001; 5: 65-69.

53 Baslow MH: Canavan's spongiform leukodystrophy: a clinical anatomy of a genetic metabolic CNS disease. J Mol Neurosci 2000; 15: $61-69$

54 Matalon RM, Michals-Matalon K: Spongy degeneration of the brain, Canavan disease: biochemical and molecular findings. Front Biosci 2000; 5: D307-D311.

55 Matalon $\mathrm{R}$ et al: Adeno-associated virus-mediated aspartoacylase gene transfer to the brain of knockout mouse for Canavan disease. Mol Ther 2003; 7: 580-587.

56 Alisky JM et al: Transduction of murine cerebellar neurons with recombinant FIV and AAV5 vectors. Neuroreport 2000; 11: 26692673.

57 Auricchio A: Pseudotyped AAV vectors for constitutive and regulated gene expression in the eye. Vision Res 2003; 43: 913-918.
58 Stopa M: Gene therapy prospects in ophthalmology. Klin Oczna 2002; 104: 289-292.

59 Martin KR, Klein RL, Quigley HA: Gene delivery to the eye using adeno-associated viral vectors. Methods 2002; 28: 267-275.

60 Fazzi E et al: Leber's congenital amaurosis: an update. Eur J Paediatr Neurol 2003; 7: 13-22.

61 Dejneka NS, Rex TS, Bennett J: Gene therapy and animal models for retinal disease. Dev Ophthalmol 2003; 37: 188-198.

62 Cremers FP, van den Hurk JA, den Hollander AI: Molecular genetics of Leber congenital amaurosis. Hum Mol Genet 2002; 11: $1169-1176$.

63 Perrault I et al: Leber congenital amaurosis. Mol Genet Metab 1999; 68: $200-208$.

64 Acland GM et al: Gene therapy restores vision in a canine model of childhood blindness. Nat Genet 2001; 28: 92-95.

65 Weber $\mathrm{M}$ et al: Recombinant adeno-associated virus serotype 4 mediates unique and exclusive long-term transduction of retinal pigmented epithelium in rat, dog, and nonhuman primate after subretinal delivery. Mol Ther 2003; 7: 774-781. 